\title{
Treatment of acquired aphasia: speech therapists and volunteers compared
}

\author{
RACHEL DAVID, PAM ENDERBY, DAVID BAINTON \\ From the Neurological/Stroke Rehabilitation Unit, Frenchay Hospital, Bristol, UK
}

SUMMARY This paper reports on a multicentre trial comparing the effects of speech therapists and untrained volunteers on recovery from aphasia following stroke. One hundred and fifty-five patients entered the study and 96 completed it. Patients in both treatment groups improved, and there were no differences overall in the amount of progress made. A small subgroup of patients who started treatment much later had equivalent initial scores and made almost as much progress as those who started earlier. It is suggested that the improvement in communication which occurred during treatment may be due both to the appropriate stimulation which was based on detailed and accurate speech therapy assessment, and to the regular support and encouragement. provided within the therapeutic relationship.

In the average District General Hospital, patients with aphasia due to stroke make up a large proportion of the speech therapy caseload. Attempts to evaluate speech therapy have been beset with methodological weaknesses, such as a high degree of patient selection,' lack of controls, ${ }^{2}$ unrealistic amounts of treatment, ${ }^{3}$ or control and treatment groups which are not comparable. ${ }^{4}$ We report here on a study which was designed to answer the question of whether any improvement relating to speech therapy was associated with the specific skills and experience of the speech therapist, or was a consequence of the general stimulation and support which most therapeutic relationships provide. To this end, we compared the outcome in two groups of patients with aphasia following stroke: one group received "conventional speech therapy" and the other received stimulation and support from untrained volunteers.

\section{Method}

The present study was a multicentre trial with fourteen participating speech therapy departments. All patients referred for speech therapy with a clinical diagnosis of stroke complicated by aphasia were eligible for the study. with the following qualifications: (1) their speech diagnosis was predominantly aphasia, (2) they had never received speech therapy at any time. (3) they were English speaking.

Address for reprint requests: Dr Rachel David. Frenchay Hospital. Bristol BSI6 ILE. UK.

Received 8 Mav 1982 and in revised form 19 July 1982. Accepted 9 August 1982
(4) they did not have associated disabilities (for example, deafness, blindness or confusion so severe that the cooperation required for assessment and treatment was not possible. (5) their aphasia was sufficiently severe to warrant treatment. This was the case when the two successive baseline assessments recorded using the Functional Communication Profile ${ }^{5}$ (see below) were less than $85 \%$.

Following referral to a speech therapy department, no patient entered the study until at least three weeks from the stroke. This was designed to allow for a large part of the spontaneous recovery that follows a stroke, and which is thought to be quite independent of formal treatment. ${ }^{67}$ There was no restriction on late referrals as in our experience a sizeable minority of patients are referred some weeks or months after a stroke. Following random allocation to their treatment group. patients in the speech therapy group were seen individually by a qualified speech therapist. who gave them such treatment as she thought appropriate for 30 hours over a period of 15-20 weeks. Volunteers were recruited by advertisement and word-ofmouth. The only requirements were that they should be able to devote two hours per week to their patient, and should be reliable. Volunteers were given a detailed description of their own patient's communication problems based on his assessment results. For example, a patient might be described in the following way: "He has a lot of difficulty in understanding what is said to him, especially if you use long sentences or change the subject quickly. His speech is limited to single words which he produces with great effort and which are not always the ones he wants to say. He has a lot of difficulty in understanding what he reads. he can only manage the headlines in the newspaper. and he is quite unable to write spontaneously, although he can copy letters and words". Volunteers were asked to encourage their patient to communicate as well as possible. They received general support, and information from the 
within-treatment assessments, but were given no guidance or instruction in speech therapy techniques. They saw the patients in the speech therapy department, and the time spent with them was the same as for the speech therapy group.

Assessments to determine the severity of the initial aphasia, the progress of the patient within-treatment, and the post-treatment outcome, were all carried out using the Functional Communication Profile-a relatively simple measure-whose validity and repeatability have been examined. Scores in five different areas of communicative function were recorded, weighted, and converted to an overall percentage of the patient's estimated previous communicative ability. Assessments for both groups of patients were made by speech therapists, who conveyed the results to the treating therapists or volunteers, and who also undertook the counselling of relatives. There were two baseline assessments, a week apart, four within-treatment assessments at 2, 4, 8 and 12 weeks from the start of treatment, and a post-treatment test immediately after the end of treatment. In the case of those patients receiving conventional speech therapy, the assessing therapist was never the treating therapist. We.reduced inter-observer variation by suitable training of the therapists as each centre was enrolled. One of us (RD) was responsible for instruction in the Functional Communication Profile and also co-ordinated the running of the trial.

\section{Results}

One hundred and fifty-five patients entered the study but 59 failed to complete it. The reasons for "dropout" are presented in Table 1 . For most causes, the number of patients lost was approximately equal in the two groups. However, the ambulance strike in the winter of 1979 caused more outpatients to be lost from the Volunteer group. A full-time speech therapist could see patients even if they turned up late or on the wrong day, whereas a volunteer might arrive at the hospital but not be able to wait indefinitely for the ambulance. The majority of withdrawn patients lapsed in the first four weeks of treatment. The patients who dropped out tended to have lower baseline scores, a longer post-onset interval and were

Table 1 Drop-outs

\begin{tabular}{|c|c|c|c|}
\hline & $\begin{array}{l}\text { Speech } \\
\text { Therapy }\end{array}$ & Volunteers & All \\
\hline Patients enrolled & 71 & 84 & 155 \\
\hline $\begin{array}{l}\text { No withdrawn during } \\
\text { Treatment }\end{array}$ & 23 & 36 & 59 \\
\hline $\begin{array}{l}\text { Drop-out rate } \\
\text { Reasons }\end{array}$ & $32 \%$ & $43 \%$ & $38 \%$ \\
\hline Death & 4 & 6 & 10 \\
\hline Further CVA & 5 & 5 & 10 \\
\hline Ambulance strikes and delays & () & 5 & 5 \\
\hline Self discharge & 2 & 6 & 8 \\
\hline Illness & 5 & 3 & 8 \\
\hline Volunteer problems & - & 4 & 4 \\
\hline Moved & 3 & 2 & 5 \\
\hline Other & 4 & 5 & 9 \\
\hline
\end{tabular}

Table 2 Background information on patients who completed treatment, and on all patients who had withintreatment assessments

\begin{tabular}{llllll}
\hline & \multicolumn{2}{c}{ Patients completing treatment } & \multicolumn{2}{c}{ All patients } \\
$N$ & $S$ & $V$ & $S$ & $V$ \\
& 48 & 48 & 65 & 68 \\
\hline Sex Men & 27 & 29 & 35 & 42 \\
Women & 21 & 19 & 30 & 26 \\
Age Mean & 70 & 66 & 70 & 65 \\
SD & $8 \cdot 9$ & $10 \cdot 1$ & $8 \cdot 7$ & $10 \cdot 6$ \\
$\begin{array}{c}\text { Weeks post-onset } \\
\text { Median }\end{array}$ & 4 & 5 & & \\
Range & $4-45$ & $4-125$ & $4-266$ & $4-432$ \\
\hline
\end{tabular}

$\mathrm{S}=$ speech therapist

$\mathrm{V}=$ volunteer

slightly younger than the patients who completed treatment.

As the main aim of the study was to describe the effects of treatment on those patients who received a full course, most of the analyses included only the 96 patients who completed treatment. However, because of the high "drop-out" rate which might have disturbed the comparability of the two treatment groups, an analysis was undertaken which excluded only the 22 patients who dropped out of the study before the first within-treatment assessment. For these subjects there was no data after the baseline tests. Table 2 shows the background details of both cohorts. The treatment groups were comparable in all aspects apart from age, where the speech therapy group was approximately five years older. The possible effect of this age difference on progress and outcome was investigated by (a) correlation Age $\times$ Baseline Functional Communication Profile scores, not significant, (b) correlation Age $\times$ Post-treatment Functional Communication Profile, not significant, (c) correlation Age $\times$ Change between baseline 2 and post-treatment 1, not significant, (d) Analysis of variance Age $\times$ Therapy $\times$ Time-no significant effect or interaction from age. It was concluded that age did not have any significant influence on the progress of the patients and in subsequent analyses it was not included as a factor. Mean Functional Communication Profile scores were calculated for the two treatment groups in both cohorts. The resulting recovery curves may be seen in fig 1 . There were no significant $t$ test differences between the treatment groups at any assessment in either analysis. Recovery in relation to the initial severity of the aphasia was investigated by dividing the patients into two groups according to the level (higher or lower than the median score) of their baseline Functional Communication Profile scores. This resulted in a High group of 22 speech therapy and 26 volunteer patients. and a Low group of 26 speech therapy and 22 volunteer patients. Change scores were calculated for each 


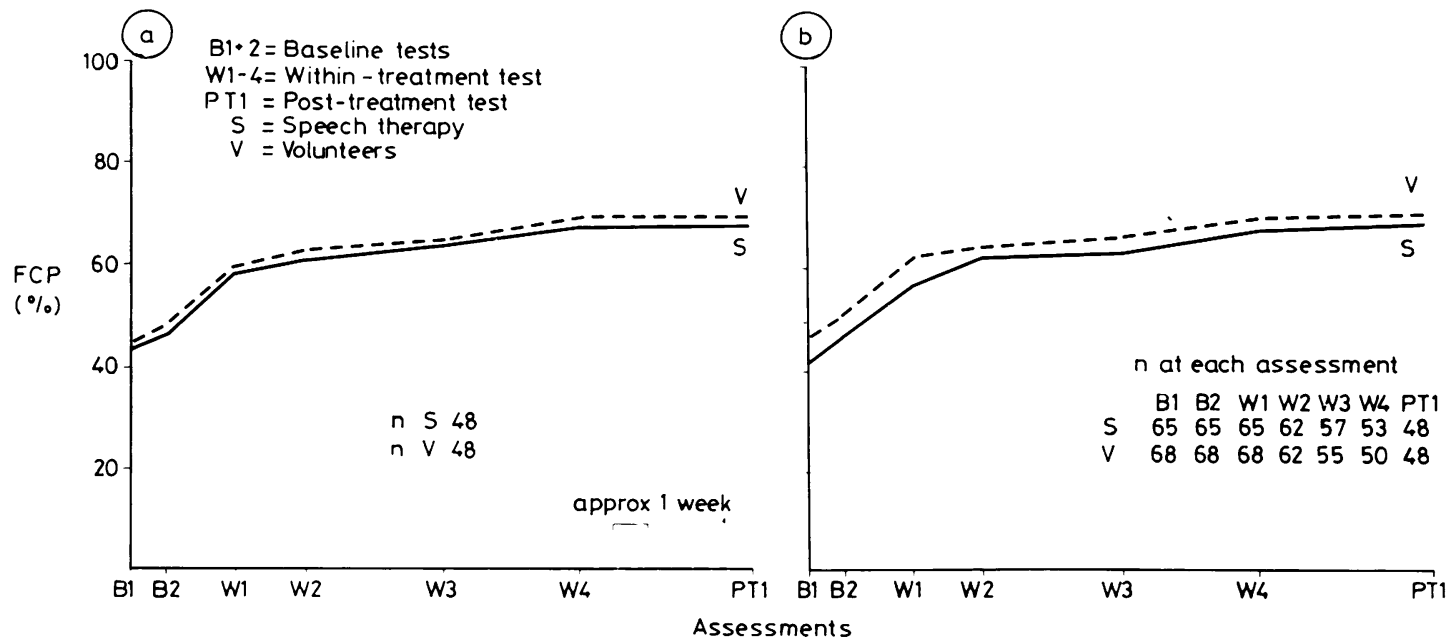

Fig 1 Mean FCP scores: speech therapy and volunteer groups (a) patients who completed treatment, (b) all patients who had within-treatment assessments

patient by taking the difference between his two baseline scores, and also the difference between the second baseline scores and that at each subsequent assessment. This procedure resulted in six scores for each patient. These were submitted to a two-way analysis of variance with repeated measures (table 3 ). This showed no significant main effects from the type of therapy or severity, and there were no significant interactions involving treatment, (that is again, no major differences were found between speech therapy and volunteer patients). The recovery curves of the four subgroups may be seen in fig 2 .

The influence of the length of the post-onset interval was also investigated. Patients were divided into three groups according to the length of the postonset interval at the beginning of treatment, and their response to treatment examined (fig 3). There were no significant $t$ test differences between the mean scores of the three groups at any assessment (table 4), and the three recovery curves were markedly similar in shape.

\section{Discussion}

We attempted to design a study which represented the kind of patients with aphasia and the amounts of treatment normally encountered in speech therapy departments in this country. This was the reason why we accepted the diagnosis of stroke without further qualifications. Our attempts to increase objectivity included random allocation of patients to the two treatment groups and our insistence that the treating therapist could not be the assessing therapist for any one patient. (Our attempt to maintain "blind" assessment, where the assessing speech therapist remained unaware of the treatment allocation of the individual patient, had failed in an earlier pilot study). ${ }^{8}$

The most important finding of this study is the evidence from the small number in our group defined as Late Referrals, that therapy has a positive effect. It is usually argued that spontaneous recovery occurs within twelve weeks. However, our Late group, where the median interval between onset of stroke

Table 3 Analysis of variance: Functional Communication Profile change scores, type of therapy and initial severity

\begin{tabular}{|c|c|c|c|c|c|}
\hline Source & $S S$ & $D F$ & $M S$ & $F$ & Sig level \\
\hline $\begin{array}{l}\text { A Therapy } \\
\text { C Severity } \\
\text { AC } \\
\text { Subjects within groups } \\
\text { B Change scores } \\
\text { AB } \\
\text { BC } \\
\text { ABC } \\
\text { B } \times \text { subjects within groups }\end{array}$ & $\begin{array}{r}38 \cdot 1 \\
1184 \cdot 9 \\
420 \cdot 7 \\
35439 \cdot 3 \\
17148 \cdot 4 \\
79 \cdot 4 \\
2491 \cdot 2 \\
572 \cdot 7 \\
29444 \cdot 4\end{array}$ & $\begin{array}{r}1 \\
1 \\
1 \\
92 \\
5 \\
5 \\
5 \\
5 \\
460\end{array}$ & $\begin{array}{r}38 \cdot 1 \\
1184 \cdot 9 \\
420 \cdot 7 \\
385 \cdot 2 \\
3429 \cdot 7 \\
15 \cdot 9 \\
498 \cdot 2 \\
114 \cdot 6 \\
64 \cdot 0\end{array}$ & $\begin{array}{r}\cdot 1 \\
3 \cdot 08 \\
1 \cdot 09 \\
58 \cdot 32 \\
\cdot 27 \\
8 \cdot 47 \\
1 \cdot 95\end{array}$ & $\begin{array}{l}\text { NS } \\
\text { NS } \\
\text { NS } \\
p<\cdot 001 \\
\text { NS } \\
p_{\text {NS }}<\cdot 001\end{array}$ \\
\hline
\end{tabular}




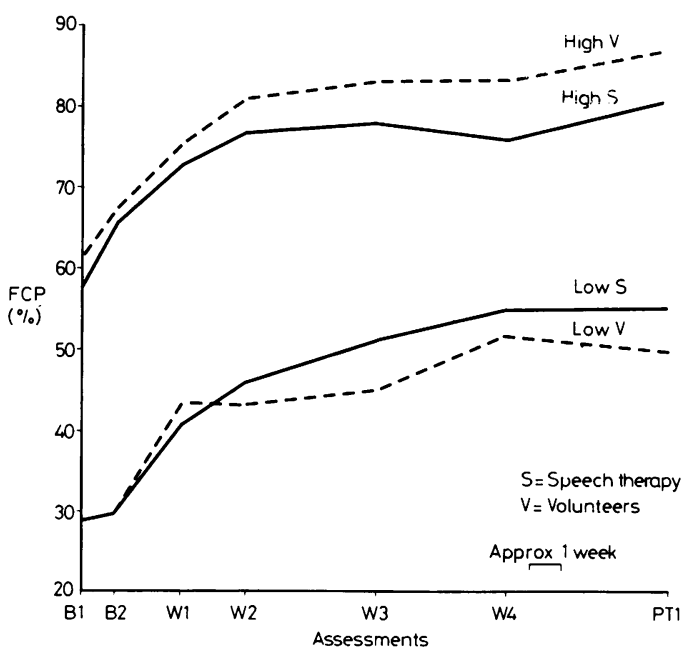

Fig 2 Mean FCP scores: High and Low Groups

and starting treatment was 20 weeks, started at a similar level to the Early and Intermediate groups and showed an almost indentical pattern of recovery. This suggests that, unless the accepted theories of recovery are to be discounted, treatment rather than spontaneous remission was responsible for most of the improvement shown by all the groups. Indeed, it appears that most of the spontaneous improvement in these patients had occurred within two months of the stroke.

If treatment works, does it matter who gives it? Both treatment groups demonstrated almost identical recovery curves (fig 1). Our evidence would suggest that a large part of the improvement associated with speech therapy for patients with stroke and complicating aphasia is the result of the interest, support and stimulation provided by speech therapist and volunteer alike when they work from a detailed assessment. Previous reports on the use of untrained volunteers have lacked controls or a second treatment group against which they could be compared. ${ }^{9}$ A report on the use of volunteers who were trained in

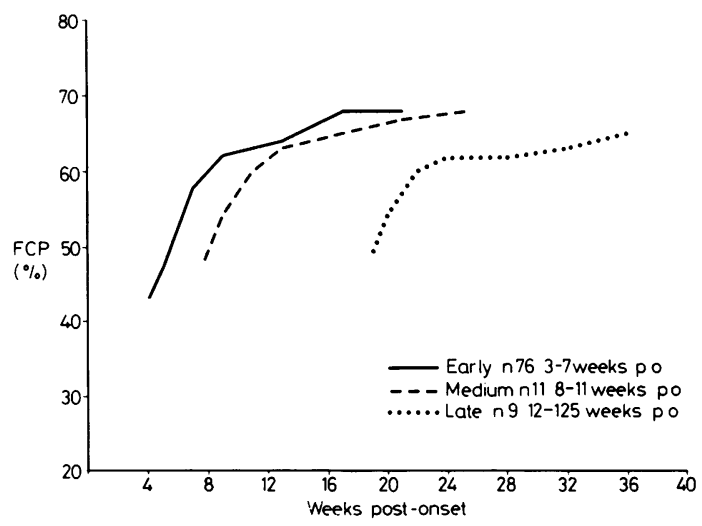

Fig 3 Mean FCP scores: Early. Medium and Late referrals

speech therapy methods showed no major differences between their results and those of speech therapists. ${ }^{10}$ On the basis of our data, any ethical doubts based on the fear that patients seen by untrained volunteers would be at a disadvantage, would appear to be groundless. However, it must be remembered that although the volunteers received no instruction in speech therapy methods, they were given the results of the regular and complete speech therapy assessments which were done on their patients, and worked within speech therapy departments. We must emphasise that our findings apply only to aphasia following stroke and cannot be extrapolated to other conditions for which speech therapy is given, nor to other types of treatment.

We are very grateful to the speech therapists who took part in this study and who were based at the following hospitals:-

Southmead Hospital, Bristol: Ashford Hospital, Middlesex; North Manchester General Hospital; St. Thomas's Hospital, London; Withington Hospital, Manchester; Horton General Hospital, Banbury; Gloucester Royal Hospital; Battle Hospital, Reading; Royal Berkshire Hospital, Reading; Victoria Infirmary, Glasgow; Odstock Hospital,

Table 4 Early, medium and late groups: mean FCP scores

\begin{tabular}{|c|c|c|c|c|c|c|c|c|}
\hline & & $B I$ & $B 2$ & $W I$ & $W 2$ & W3 & W4 & PTI \\
\hline Early & Mean & $43 \cdot 1$ & $46 \cdot 8$ & $57 \cdot 8$ & $62 \cdot 0$ & $64 \cdot 2$ & 67.8 & 68.2 \\
\hline \multirow[t]{2}{*}{$N=76$} & SD & 20.1 & $20 \cdot 6$ & $20 \cdot 4$ & $22 \cdot 0$ & $22 \cdot 2$ & $20-3$ & 21.6 \\
\hline & & $2 \cdot 3$ & $2 \cdot 4$ & $2 \cdot 4$ & $2 \cdot 5$ & 2.6 & $2 \cdot 3$ & 2.5 \\
\hline \multirow{3}{*}{$\begin{array}{l}\text { Medium } \\
N=11\end{array}$} & Mean & $48 \cdot 2$ & $52 \cdot 8$ & $60 \cdot 1$ & $63 \cdot 0$ & 66.8 & $70 \cdot 4$ & 70.7 \\
\hline & SD & 15.5 & $20 \cdot 3$ & 17.8 & 19.8 & 18.6 & $16 \cdot 3$ & 18.7 \\
\hline & SE & $4 \cdot 8$ & $6 \cdot 3$ & $5 \cdot 6$ & $6 \cdot 2$ & 5.8 & $5 \cdot 1$ & 5.8 \\
\hline \multirow{3}{*}{$\begin{array}{l}\text { Late } \\
N=9\end{array}$} & Mean & $49 \cdot 1$ & 54.5 & $59 \cdot 9$ & $62 \cdot 0$ & $62 \cdot 3$ & $62 \cdot 8$ & 64.6 \\
\hline & SD & $27 \cdot 0$ & $23 \cdot 3$ & $23 \cdot 4$ & 21.9 & $22 \cdot 6$ & $25 \cdot 1$ & $22 \cdot 4$ \\
\hline & SE & $9 \cdot 6$ & $8 \cdot 3$ & 8.4 & 7.8 & $8 \cdot 1$ & $9 \cdot 0$ & $8 \cdot 0$ \\
\hline
\end{tabular}

No $t$ test values significant between groups 
Salisbury; Blackburn Royal Infirmary; Cossham Hospital, Bristol; Frenchay Hospital, Bristol.

We thank the Department of Health and Social Security for funding, and Peter Sweetnam for statistical advice.

\section{References}

' Hagen C. Communicative abilities in hemiplegia: effect of speech therapy. Arch Phys Med Rehabil 1973;54: 454-63.

2 Sands E, Sarno MT, Shankweiler D. Long term assessment of language function in aphasia due to stroke. Arch Phys Med Rehabil 1969;50:202-7.

${ }^{3}$ Smith A. Diagnosis, intelligence and rehabilitation of chronic aphasics. Ann Arbor: University of Michigan Department of Physical Medicine and Rehabilitation 1972.
+ Basso A. Capitani E, Vignolo LA. Influence of rehabilitation on language skills in aphasic patients: a controlled study. Arch Neurol 1979;36:190-6.

5 Sarno MT. The Functional Communication Profile: manual of directions. Rehabilitation Monograph 42; New York Institute of Rehabilitation Medicine, 1969.

- Culton GL. Spontaneous recovery from aphasia. $J$ Speech Hearing Res 1969;12:825-32.

7 Sarno J, Sarno M, Levita E. Evaluating language improvement after completed stroke. Arch Phys Med Rehabil 1971;52:73-8.

${ }^{8}$ David RM, Enderby P, Bainton D. Progress report on an evaluation of speech therapy for aphasia. Br J Disord Commun 1979;14(2):85-8.

${ }^{9}$ Eaton Griffith V, Miller C. Volunteer stroke scheme for dysphasic patients with stroke. $\mathrm{Br}$ Med J 198();281: 1605-8.

${ }^{10}$ Meikle M, Wechsler E, Tupper A, et al. Comparative trial of volunteer and professional treatments of dysphasia after stroke. Br Med J 1979;2:87-9. 